\title{
DELI: An interactive new product development tool for the analysis and evaluation of market research data
}

Received (in revised form): 4th March, 2003

\section{Martin Natter}

is Associate Professor at the Wirtschaftsuniversität Wien, Austria. His previous research has appeared in Management Science, Marketing Letters, Journal of Retailing and Consumer Services and others. His major research interests lie in the fields of new product development, market segmentation and pricing.

\section{Andreas Mild}

is Assistant Professor at the Wirtschaftsuniversität Wien, Austria. His previous research has appeared in MIS Quarterly, Management Science, Journal of Retailing and Consumer Services and others. His major research focuses on knowledge discovery techniques, agent-based simulation and new product development.

Martin Natter and Andreas Mild Department for Production Management, Vienna University of Economics \& BA, Pappenheimgasse 35/3/5, A-1200 Vienna, Austria.

Tel: +431313365613 ; Fax: +431313365610 ; e-mail:

martin.natter@wu-wien.ac.at andreas.mild@wu-wien.ac.at

\begin{abstract}
This paper presents DELI, a new interactive tool for supporting new product development decisions. DELI addresses the 'chicken and egg' problem in new product development: a product's features shape the way that the market is segmented and targeted, but that very segmentation/targeting itself determines which features the product needs to incorporate. It is very useful, therefore, to be able to look at attributes, product positions and segments in a 'single hit' to measure the key trade-offs available. DELI integrates segmentation, visualisation of competitive structures and the segment-specific identification of new product functionality. Several interactive features support the search for new products. Furthermore, the authors introduce a novel conditional segmentation, mapping and positioning approach for an improved representation of products and customers within one map, supporting interpretation and segment-specific new product development.
\end{abstract}

\section{INTRODUCTION}

In early phases of new product development, management faces the challenge to select from a wide variety of potential feature attributes or product benefits to be included in a new product concept. In order to extract the right features, market research, in particular conjoint analysis, is used to measure customers' preferences. Conjoint analysis aims at identifying the most important attributes for customers in order to find (segment-specific) optimal product configurations. ${ }^{1-3}$ Although there are several software solutions for conducting conjoint analyses and estimating parameters, these tools provide poor graphical support, focusing mainly on simulating shares-of-preference for well-defined scenarios.

Adaptive conjoint analysis, ${ }^{4}$ one of the most frequently applied conjoint tools, allows the incorporation of more attributes than other approaches (eg 
choice-based conjoint analysis). ${ }^{5}$ For designing the conjoint analysis, however, one has to collect knowledge about the most interesting benefits or new product features and customer requirements. When the number of attributes and levels is exhaustive, the required interview time and the number of interviews become prohibitive even for adaptive conjoint analysis. Therefore, a conjoint analysis often follows a more general market research study in which the relevant product attributes, benefits or shortcomings of existing products are identified. The approach presented in this paper aims at supporting this task. In this early development phase, new product developers are interested in questions of the following type.

- What are the interesting new features or benefits for a new product?

- How do specific customer segments assess such new features?

- How well do existing products satisfy needs and wants in different segments?

- What are the most important attributes for a segment?

- Are there segments which are not (sufficiently) served with existing products?

- What do segment-specific bundles of attributes or product functionality look like?

In addition, to achieve breakthrough innovations, new product development (NPD) teams can no longer be limited to different marketing functions but include people with different business orientations (eg engineering, IT, finance, manufacturing) requiring the use of tools that are intuitive and produce results that are easy to communicate. The advantage of the application of integrative tools for NPD has been demonstrated theoretically ${ }^{6,7}$ and empirically. ${ }^{8}$ In this paper, the authors present DELI, a new tool that combines multidimensional scaling (MDS), ${ }^{9,10}$ customer segmentation and joint-space mapping to support the early product development phase. The main advantage lies in its intuitive graphical presentation, the possibility of using many potential product features and an improved approach for the simultaneous presentation of customers, products and attributes within one map.

\section{THE DELI APPROACH}

Lilien and Rangaswamy ${ }^{11}$ propose a three-step process for developing marketing strategies, consisting of segmentation, targeting and positioning (STP). The purpose of segmentation is to find an optimal number of homogenous market segments in terms of customer needs, wants, benefits sought, problem solutions desired and usage situations, and to describe these segments by variables (spending power, price sensitivity) which help the firm to understand how to serve these segments and how to talk to these customers (media preferences, opinions, interests, etc). The targeting step is concerned with the evaluation of segments (attractiveness, growth rate, distribution costs) and the selection of one or more target segments. Positioning aims at finding products and services that attract the targeted customers. Customer perceptions and preferences for a set of alternatives are typically presented on a map in Euclidean space using multidimensional scaling methods. For practical application, this process should be extended by an evaluation or market simulation step $^{12}$ which triggers an interactive iterative development process. DELI builds on this extended STP approach, supporting the graphical intuition of new product developers.

In accordance with the proposed STP process, in the first step, customers are 


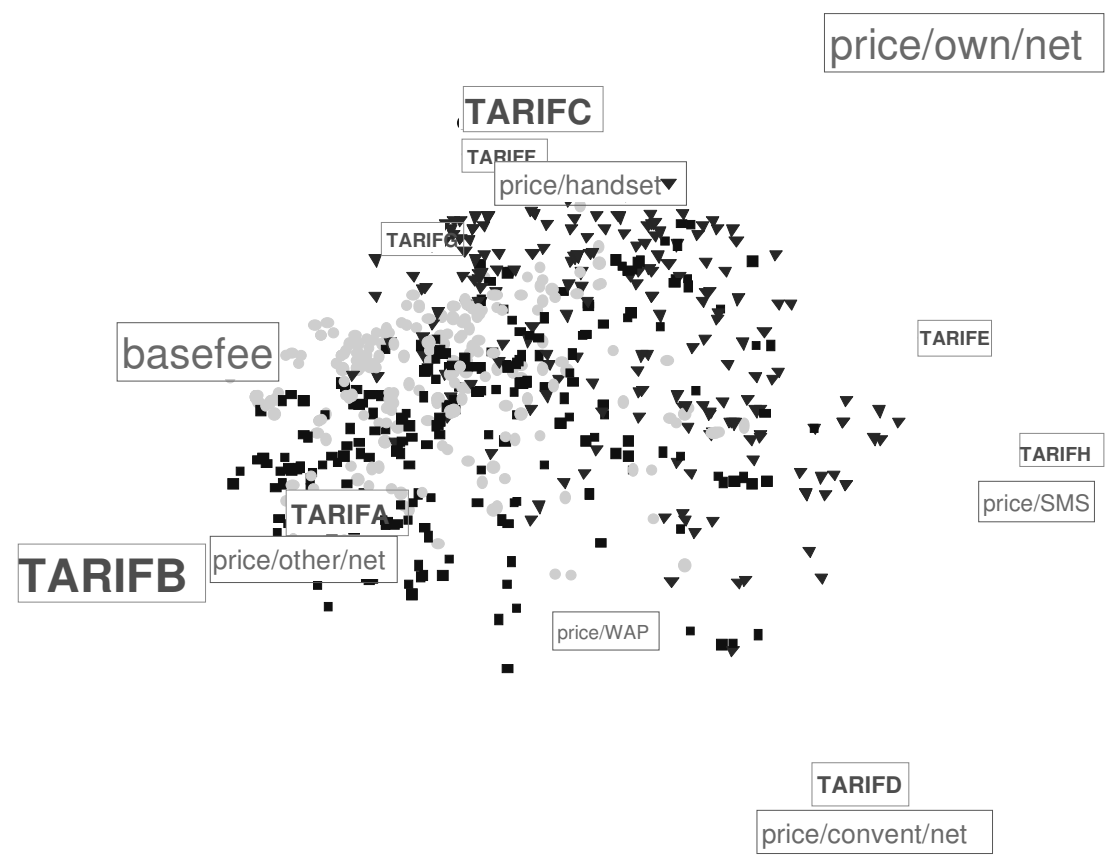

Figure 1 Map based on the consecutive segmentation-MDS-positioning approach showing attribute (lower case), product (in capital letters) and customer positions (segment-specific symbols)

segmented into homogeneous groups using the $\mathrm{k}$-means clustering algorithm. ${ }^{13}$ The optimal number of segments is determined using the Davies-Bouldin Index. ${ }^{14}$ In the second step, an initial MDS solution is computed based on distances between product and attribute ratings/rankings. In the third step, customers are positioned in the map according to their individual preferences towards all assessed products and attributes. The customer position is calculated as a weighted average over all product and attribute positions using the importance customers place on them as weighting factors. A typical result of a consecutive application of clustering and MDS is shown in Figure 1. Since the MDS solution is not conditional on the segment solution derived in the first step, customer positions and segment memberships do not match. As this joint presentation of products/attributes and customers prevents a segment-specific interpretation and analysis, such a solution is of hardly any use for practical product development. In the marketing literature, benefits of simultaneous approaches have been demonstrated for various applications. ${ }^{15-17}$ Therefore, this consecutive approach has been replaced by a conditional segmentation, multidimensional scaling and customer positioning approach in the following way:

— initial k-means solutions (several replications with different starting solutions) are computed and the optimal number of segments is selected (Davies-Bouldin Index);

- the MDS solution is computed using an extended distance matrix including the segment membership vectors;

- customers are positioned in the map (as described above);

- a new segment solution is derived using an extended segmentation base including the customer positions as additional segmentation criteria. In 


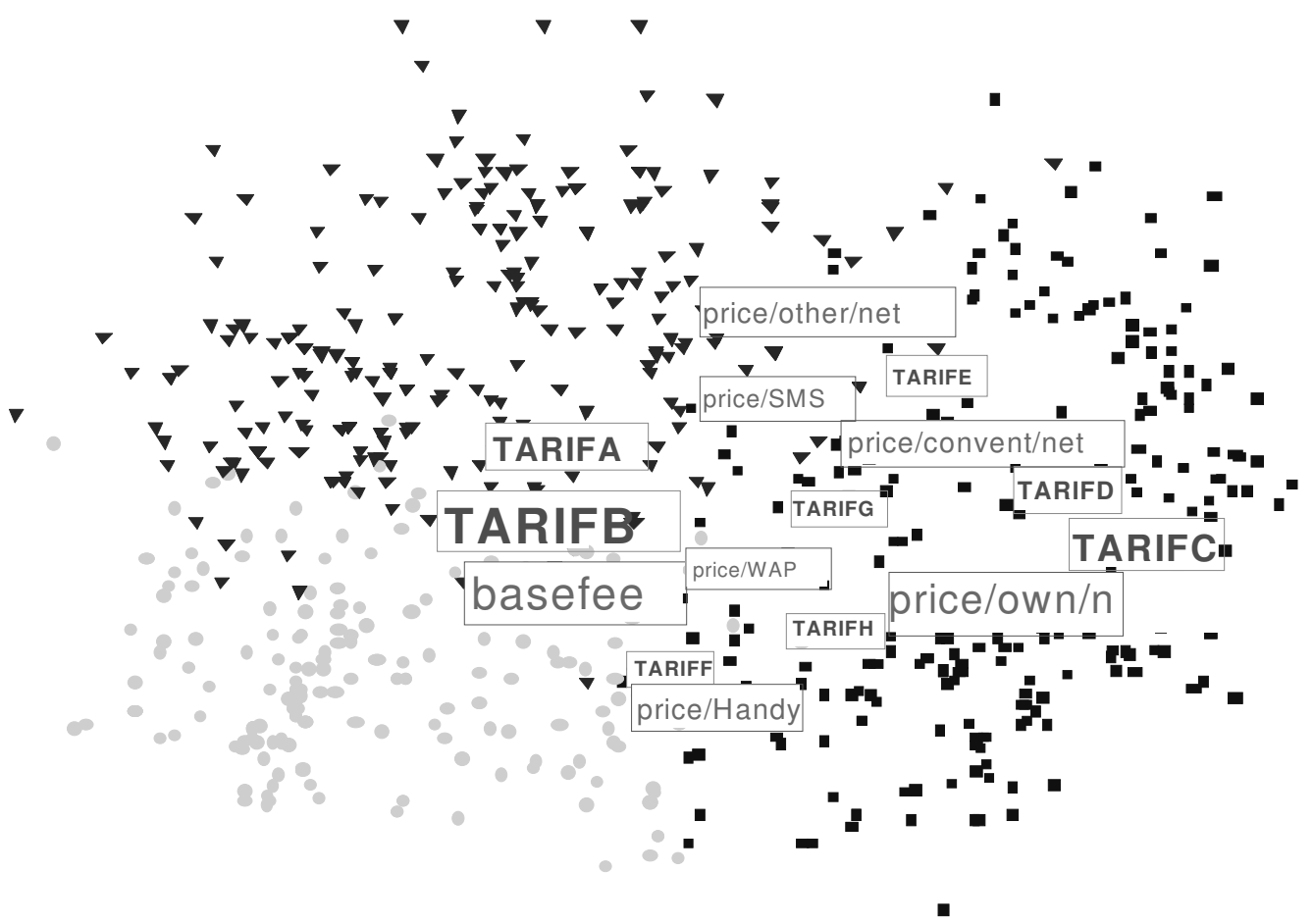

Figure 2 Map based on the conditional segmentation-MDS-positioning approach showing attribute (lower case), product (in capital letters) and customer positions (segment-specific symbols)

this new approach, the segment memberships influence the attribute and product positions in the map, which in turn determine the customer positions. In DELI, steps $2-4$ are iterated until convergence is achieved.

In a final step,

- positions of products and attributes are calculated based on a weighted average of the customer positions. The weights are determined based on the product and attribute preference of each customer.

Figure 2 demonstrates the improved outcome. It can be seen that the segment overlap is reduced considerably, enabling direct interpretation and segment specific analysis of the market. Relative distances between customer positions and product/attribute positions play the key role when such maps are interpreted. In order to test whether the reduced segment overlap has a significant negative impact on the information the new map provides, the information content of the two maps were compared based on a model with product/attribute specific preferences as dependent variables and distances between customer positions and product/attribute positions as predicting variables. The average correlation between actual and predicted ranks is $r=66$ per cent for the configuration shown in Figure 1 and $r=63$ per cent for the configuration shown in Figure 2. Given this moderate loss in terms of correlation one can easily justify the increased usability of the graphical outcome.

\section{Data requirements}

The minimum questionnaire design for DELI requires the six types of question shown in Table 1. 
Table 1: Minimum design of questionnaires for using DELI

\begin{tabular}{llc}
\hline Question & Example & Data field \\
\hline 1. Rank (or rate) products & Tariff A & 2 \\
& Tariff B & 1 \\
& Tariff C & 3 \\
2. Rank (or rate) attributes & Base fee per month & 1 \\
& Provider & 3 \\
3. Rank (or rate) benefits & Price into other networks & 2 \\
& Free cine card/quarter & 2 \\
4. Rank (or rate) levels of all non-metric and & Free coffee & 3 \\
non-binary (yes/no) attributes & Free magazine & 3 \\
5. Rank (or rate) levels of all non-metric and & Provider A & 2 \\
non-binary (yes/no) benefits & Provider B & 1 \\
6. Expenditures in that product category & Provider C & 1 \\
\hline
\end{tabular}

Variables generated by these six types of question are necessary in order to derive the maps and use the market simulation. Market research for new product decisions should, however, besides the usual battery of questions concerning demographics, lifestyle, media and image, collect more encompassing product specific information such as:

1 disturbing factors of current products

2 usage situations: how is the product used?

3 awareness

4 buying behaviour

5 switching behaviour.

Additional information from 1-2 can provide useful information on innovative new features or product improvements. Data on 3 can help to identify problems in communication. Data on 4-5 can support the selection of the target group. When the number of attributes/benefits is not exhaustive, choice-based conjoint interview data can also be used in DELI for market simulation. Questionnaire design for internet or computer-based interviews is supported outside DELI with another tool (not described in detail here). DELI can import whole questionnaires, however, considerably reducing the usual effort of encoding and transforming raw data into an appropriate format.

\section{CASE STUDY}

The approach is demonstrated using a market research survey in the telecommunications sector. The survey contains 738 interviews where customers are asked to provide ratings/rankings of existing products and concepts (TarifA, TarifB ...); product attributes (base/fee/month, price/ conventional/network, price/other/ network, price/SMS/, price/WAP, price/handset, price/own/net); as well as demographic (age, income, sex); and lifestyle (leisure preferences) data. Furthermore, the respondents had to rate a wide variety of potential new benefits or applications. The aim of this study was to define new product concepts for different target groups and to evaluate the attraction of these concepts.

Figure 2 demonstrates the application of the joint mapping of attributes/ products and consumers: all products or product concepts (in black) are positioned according to their perceived 


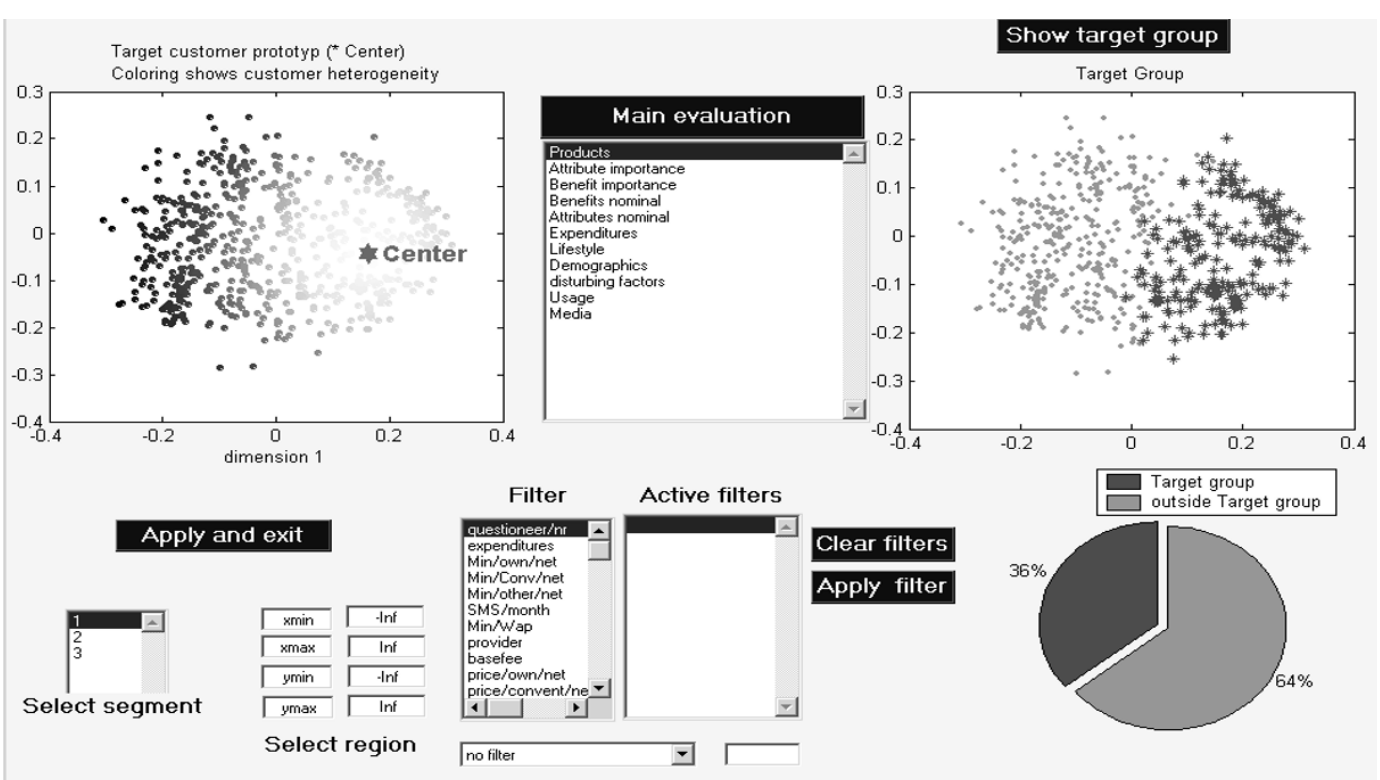

Figure 3 Selection of the target group and evaluations

difference. Similarly perceived products are positioned close to each other. The relative distance between products indicates the strength of competition. TarifA, for example, is competing with TarifB, whereas TarifC competes with TarifD. The size of the product label indicates the overall attractiveness of a product, with bigger size indicating higher attractiveness. For example, TarifB is preferred to TarifA by a majority.

Attributes (grey labels) are positioned in the same way as products. When two attributes are located close to each other, customers rate both attributes as important or both as unimportant indicating attribute bundles for new products. Attributes located close to a product play an important role for customers in that area. The bigger the size of the attribute label, the higher the overall importance of this attribute. For example, customers choosing TarifD judge the price for calling into a conventional network as important, whereas customers of TarifB focus on the monthly base fee. Every customer is displayed as a point in the map, the shade signifying the segment to which the customer belongs. Small distances between a product and a customer position indicate high preference for this product. Accordingly, lower distances between customer position and attribute position point to a higher importance of the attribute for the customer, ie customers in segment 1 (squares) prefer TarifC and TarifD whereas customers in segment 2 (triangles) and 3 (dots) have the highest preference for TarifA and TarifB.

Figure 3 shows the selection interface for the target group. The target group can be defined on the basis of segment membership, regional restrictions in the map, and any combination of filters on variables (eg income $>2000$ ). In the example, the target group is defined on the basis of the segment membership (segment 1). The pie chart indicates that 36 per cent of the initial population belongs to the target group. The scatter plot on the right hand side highlights (stars) the selected customers. The plot 


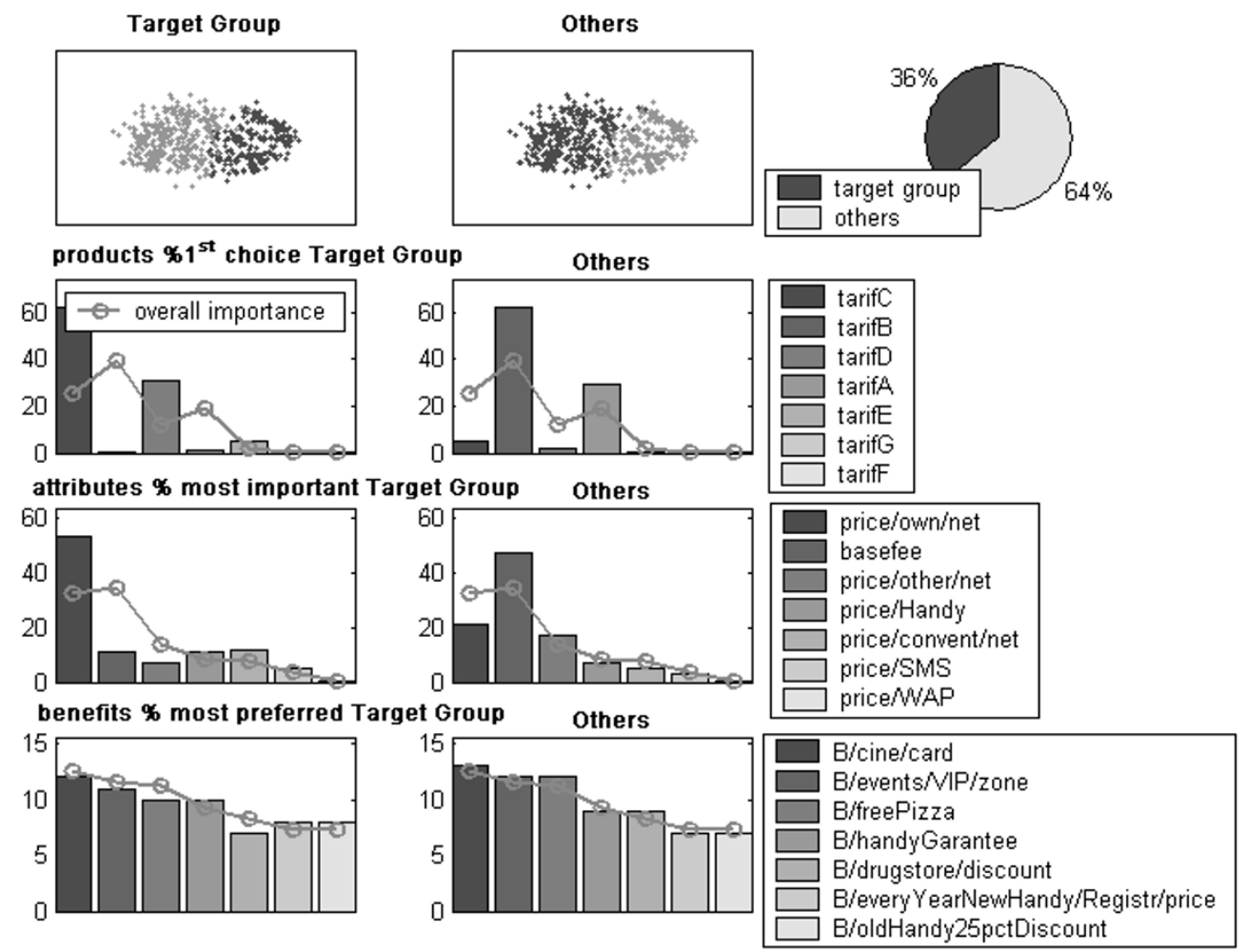

Figure 4 Analysis of the target group (segment 1) versus others. The figure shows the selected customers on the map (dark dots), the relative size of the selected target group, and the top seven products, attributes and benefits in terms of percentages of top ranks over all customers. The line indicates the overall importance

on the left hand side shows the map including the product attributes and the centre of all selected customers. The shade of the individual customers indicates the heterogeneity of their preference for a hypothetical customer prototype (the closer the customers are located to the centre, the greater their similarity). The list box topped by the 'Main evaluation' button in the centre offers evaluations of the target group in terms of the most preferred products and attributes (shown in Figure 4), as well as any question from the questionnaire (eg buying behaviour, benefits and disturbing factors, lifestyle and demographics).

As an example, the development of a new product concept for segment 1 is shown. Figure 4 gives an overview of the preference structure for the defined target group and compares it to customers outside the target group. It can be seen that products TarifC, TarifD and TarifE are the most preferred products in the target group. The most important attributes for the target group are the price paid for calling a phone in the same network, the price paid for calling the conventional network, and the base fee per month. While price paid for calls in the same network and price for calls in the conventional network are typical order winners for segment 1 , base fee per month plays a dominant role for all segments (see Figure 2). A new product for this target group should be defined in accordance with the attribute importance shown in 
Figure 4. In the next step, there is a description of the way in which products or new concepts are evaluated.

Due to the typically very high number of potential benefits and attributes in the case of a new market entry, the authors recommend the use of an individual level scoring model for the evaluation of products. In a study published in 2000, Hensel-Börner and Sattler ${ }^{18}$ found empirically that simple compositional approaches were not clearly outperformed by adaptive conjoint analysis. For a more accurate market simulation in later phases of NPD, however, companies usually rely on more advanced approaches such as Hierarchical Bayes or finite mixture conjoint analysis models. ${ }^{19}$ Here, the score for customer $i$ on product $j$ is determined by the importance customer $i$ places on attribute $k, w_{i, k}$, and the relative attractiveness of the chosen level of attribute $k$ and product $j(j=1, \ldots, J)$ :

$$
y_{i, j}=\sum_{k} w_{i, k} x_{j, k}
$$

The importance weights for all attributes can be derived directly from the questionnaire. In the case of rankings, the data are transformed into preferences. The relative attractiveness $x_{j, k}$ of an attribute level, $v_{j, k}$, depends on the range of the feature levels of all existing products or concepts in the following way:

$$
x_{j, k}=\frac{v_{j, k}-\min _{l=1}^{J}\left(v_{l}\right)}{\max _{l=1}^{J}\left(v_{l}\right)-\min _{l=1}^{J}\left(v_{l}\right)}
$$

In the case of decreasing utility with increasing levels (eg price), $x_{j, k}$ is re-scaled by $x_{j, k}=1-x_{j, k}$. Thus, in this vector model, the score of a product is always dependent on the weights customers place on the individually assessed attributes and the relative attractiveness of the product levels. In contrast to part-worth utilities which are assigned to the according levels, an advantage of this approach lies in the fact that only the range of an attribute has to be known. This advantage is mainly due to the high uncertainty managers face in early NPD phases.

The average importance of each attribute for the targeted group serves as an indicator for order-winning product features (line in Figure 5). The overall attractiveness of a product for a target group is determined on the basis of the maximum score the consumers place on the defined products. The aggregation of the maximum scores over all customers in the target group serves as an indicator of the products' attractiveness (pie chart). In this example, it can be seen that 41 per cent of the customers in the target group attach the highest score to the new product (newtarif).

When the development team has found a satisfying set of concepts for the relevant target groups, these concepts can serve as a valuable input for a subsequent (prototype supported) conjoint analysis. Since this approach helps to identify the most relevant product features and target group characteristics, the results of the conjoint analysis will deliver more accurate and reliable results with reduced interviewing effort due to the reduced number of parameters to be estimated. When a conjoint analysis has been conducted in later stages of the NPD process (or when only smaller numbers of attributes are under investigation), the estimated utilities can also be used for DELI in order to analyse the market and receive appropriate visualisations. Of course, the simple scoring model can then be replaced with the appropriate market simulation model. For this case, DELI supports segment (or target group) specific estimation of choice-basedconjoint models including scenario definition and market simulation. 

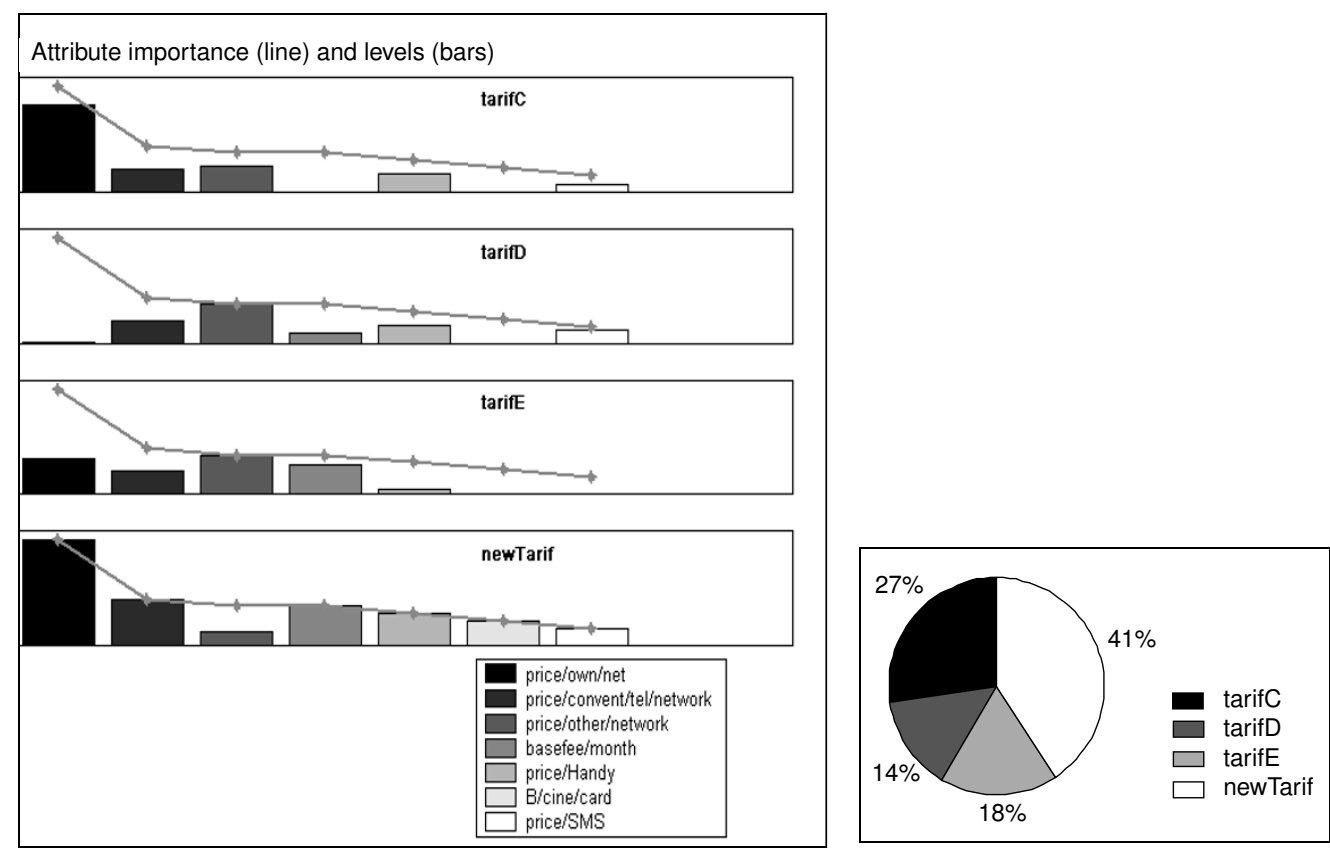

Figure 5 Bar charts for each product indicating order-winning attributes. The line shows the maximum impact of each feature according to the preferences of the defined target group. The pie chart at the right hand side shows the predicted shares-of-preference for the target group and the defined scenario

\section{SUMMARY AND CONCLUSION}

The authors have presented DELI, an interactive tool that assists new product managers in early phases of market analysis. DELI uses a market survey as input where customers rate or rank products and features. In contrast to standard market research methodologies such as conjoint analysis, this approach is able to cope with a large number of potential product features and levels. Its main advantages lie in the integration of segmentation, visualisation, new product definition and market simulation. Based on an integrated view of products, attributes and customers, new product managers are able to learn about competition, feature bundles and segment structure. This view supports the definition of the target group and the identification of potentially interesting features on which to focus. The authors have introduced a novel conditional segmentation, mapping and positioning approach for an improved representation of products and customers within one map, supporting interpretation and segment-specific NPD. DELI may be of limited use, however, when the target group is already defined prior to market research and interviews are only collected from respondents belonging to this target group. Future research could compare the usefulness of alternative clustering or scaling approaches for deriving integrative maps. Long-term investigations on real product introductions could also externally validate the predictive power of DELI.

\section{Acknowledgment}

The authors would like to express their gratitude to the editor and two anonymous referees for valuable comments and suggestions on a previous version of this paper. Furthermore, they would like to thank Christian Geberth, Alfred Taudes, Walter Aigner, Peter Cilek and Martin Ruckensteiner for valuable inputs. 


\section{References}

1 Baier, D. and Säuberlich, F. (1997)

'Kundennutzenschätzung mittels individueller Hybrid-Conjointanalyse', Zeitschrift für betriebswirtschaftliche Forschung und Praxis, Vol. 49, pp. 951-972.

2 Bauer, H. H., Hermann, A. and Gutsche, J. (1995) 'Grundprobleme und Perspektiven einer gewinnmaximalen Produktgestaltung mittels des Conjoint+Cost-Ansatzes', Zeitschrift für Betriebswirtschaft, Vol. 65, No. 12, pp. 1443-1451.

3 Steiner, W. and Hruschka, H. (2002) 'Produktliniengestaltung mit genetischen Algorithmen', Zeitschrift für betriebswirtschaftliche Forschung und Praxis, Vol. 54, No. 11, pp. 575-602.

4 Green, P. E., Krieger, A. M. and Agarwal, M. K. (1991) 'Adaptive conjoint analysis: Some caveats and suggestions', Journal of Marketing Research, Vol. 28, No. 2, pp. 215-222.

5 Natter, M. and Feurstein, M. (2002) 'Real world performance of choice-based conjoint models', European Journal of Operational Research, Vol. 137, pp. 448-458.

6 Hauser, J. R. and Clausing, D. (1988), 'The House of Quality', Harvard Business Review, pp. 63-73.

7 Natter, M., Mild, A., Feurstein, M., Dorffner, G. and Taudes, A. (2001) 'The effect of incentive schemes and organizational arrangements on the new product development process', Management Science, Vol. 47, No. 8, pp. 1029-1045.

8 Hauser, J. R. (1993) 'How Puritan-Bennett used the House of Quality', Sloan Management Review, Vol. 34, No. 3, pp. 61-70.

9 Carroll, J. D. and Arabie, P. (1980) 'Multidimensional scaling', in Rosenzweig, M. R. and Porter, L. W. (eds) Annual Review of Psychology, Vol. 31, pp. 607-649.

10 Green, P. E. and Carmone, F. J. (1972) 'Multidimensional scaling and related techniques in marketing analysis', Allyn \& Bacon, Boston, Massachusetts.
11 Lilien, G. and Rangaswamy, A. (1997), 'Marketing engineering: Computer assisted marketing analysis and planning', Addison Wesley, New York.

12 Albers, S. and Brockhoff, K. (1985), 'Die Gültigkeit der Ergebnisse eines Testmarksimulators bei unterschiedlichen Daten und Auswertungsmethoden', Zeitschrift für betriebswirtschaftliche Forschung und Praxis, Vol. 37, pp. 191-217.

13 McQueen, J. B. (1967) 'Some methods of classification and analysis of multivariate observations', in Le Cam, L. M. and Neyman, J. (eds) 'Proceedings of Fifth Berkeley Symposium on Mathematical Statistics and Probability', pp. 281-297.

14 Davies, D. L. and Bouldin, D. W. (1979) 'A cluster separation measure', IEEE Transactions on Pattern Analysis and Machine Intelligence, Vol. 1, No. 2, pp. 224-227.

15 DeSarbo, W. S., Howard, D. J. and Jedidi, K. (1991) 'MULTICLUS: A new method for simultaneously performing multidimensional scaling and cluster analysis', Psychometrika, Vol. 56, pp. 121-136.

16 Natter, M. (1999) 'Conditional market segmentation by neural networks: A Monte Carlo study', Journal of Retailing and Consumer Services, Vol. 6, No. 4, pp. 237-248.

17 Reutterer, T. and Natter, M. (2000) 'Segmentation based competitive analysis with MULTICLUS and topology representing networks', Computers and Operations Research, Vol. 27, pp. 1227-1247.

18 Hensel-Börner, S. and Sattler, H. (2000) 'Ein empirischer Validitätsvergleich zwischen der Customized Computerized Conjoint Analysis (CCC), der Adaptive Conjoint Analysis (ACA) und Self-Explicated-Verfahren', Zeitschrift für Betriebswirtschaft, Vol. 70, pp. 705-727.

19 Andrews, R. L., Ansari, A. and Currim, I. (2002) 'Hierarchical Bayes vs. Finite mixture conjoint analysis models: A comparison of fit, prediction, and partworth recovery', Journal of Marketing Research, Vol. 39, No. 1, pp. 87-98. 